Review began 02/02/2022 Review ended 02/19/2022 Published 02/25/2022

๑) Copyright 2022

Shankar et al. This is an open access article distributed under the terms of the Creative Commons Attribution License CCBY 4.0., which permits unrestricted use, distribution, and reproduction in any medium, provided the original author and source are credited.

\section{An Evaluation of the Predictive Value of Sepsis Patient Evaluation in the Emergency Department (SPEED) Score in Estimating 28-Day Mortality Among Patients With Sepsis Presenting to the Emergency Department: A Prospective Observational Study}

Takshak Shankar $^{1}$, Nidhi Kaeley ${ }^{1}$, Vempalli Nagasubramanyam ${ }^{1}$, Yogesh Bahurupi ${ }^{2}$, Archana Bairwa ${ }^{1}$, D J L. Infimate ${ }^{1}$, Reshma Asokan ${ }^{1}$, Krishna Shukla ${ }^{1}$, Santosh S. Galagali ${ }^{1}$

1. Emergency Medicine, All India Institute of Medical Sciences, Rishikesh, Rishikesh, IND 2. Community \& Family Medicine, All India Institute of Medical Sciences, Rishikesh, Rishikesh, IND

Corresponding author: Nidhi Kaeley, drnidhi_kaeley@yahoo.com

\section{Abstract}

\section{Background and objective}

Sepsis is a life-threatening medical emergency and a significant cause of mortality. Risk stratification scores for sepsis can be unsuitable for use in the emergency department (ED) due to their complexity, and an appropriate solution has yet to be found. In this study, the predictive value of the Sepsis Patient Evaluation in the Emergency Department (SPEED) score in estimating 28-day mortality was assessed among patients with sepsis presenting to the ED, in order to determine its suitability as an efficient risk stratification system.

\section{Materials and methods}

This was a single-center, prospective observational study conducted at an urban tertiary care center. We included patients presenting to the ED with suspected or confirmed sepsis who met the inclusion and exclusion criteria of our study. The patients were evaluated with the following scoring systems on arrival: the SPEED score; Predisposition, Infection, Response, and Organ dysfunction (PIRO) score; and Mortality in Emergency Department Sepsis (MEDS) score; the patients were subsequently followed up on the 28th day to record the final outcomes with regard to mortality and discharge rates.

\section{Results}

This study included 127 patients in total. The median age of the study population was 49 years, and the 28 day mortality rate was $50.4 \%$. The area under the receiver operating characteristic (AUROC) curve for the SPEED score for predicting mortality was 0.899 (95\% CI: 0.847-0.951). In comparison, the AUROC for MEDS and PIRO scores was 0.857 (95\% CI: 0.793-0.92) and 0.895 (95\% CI: 0.838-0.951), respectively. Based on the DeLong test, no significant difference was found in the diagnostic performances with respect to these scores.

\section{Conclusion}

The SPEED score is a simple and handy parameter that can be used for the early and appropriate risk stratification of patients with sepsis in the ED.

Categories: Emergency Medicine

Keywords: 28-day mortality, piro score, meds score, speed score, sepsis

\section{Introduction}

Despite the advances in modern antibiotics and resuscitation measures, sepsis remains a major cause of morbidity and mortality worldwide. In 2017, there were an estimated 48.9 million cases of sepsis. Globally, there were 11 million sepsis-related deaths, representing around $19.7 \%$ of all deaths worldwide [1]. In India, patients with sepsis and septic shock have a reported mortality rate of $35.8 \%$ and $63.6 \%$, respectively, making sepsis a significant burden on the Indian healthcare system [2].

According to the SEPSIS-3 guidelines of 2016, sepsis is a life-threatening organ dysfunction caused by the dysregulated, overactive host response to an infection. An increase in the Sequential Organ Failure Assessment (SOFA) score of $\geqslant 2$ points qualifies as organ dysfunction. Septic shock is a subtype of sepsis, 
where the underlying circulatory and cellular/metabolic abnormalities are profound enough to significantly increase mortality. Clinically, it is diagnosed by the presence of sepsis and the requirement of vasopressor therapy to elevate MAP (Mean Arterial Pressure) $\geqslant 65 \mathrm{~mm} / \mathrm{Hg}$ and lactate levels $>2 \mathrm{mmol} / \mathrm{L}(18 \mathrm{mg} / \mathrm{dL}$ ) despite adequate fluid resuscitation [3].

In order to reduce mortality related to sepsis, early detection and aggressive treatment of patients are essential $[4,5]$. Patients with sepsis usually present to the emergency department (ED) initially for evaluation. While these patients may not appear too ill at initial presentation, their condition can deteriorate rapidly. Accurate assessment of the severity and risk for mortality at initial presentation becomes essential in the ED, to differentiate patients who require intensive care from patients who can be managed in the wards. Given the typical time constraints in the ED, these decisions about patient management and disposition need to be made quickly and efficiently. Patients who are transferred to an ICU directly from the ED have lower mortality rates than those who need to be shifted to an ICU from the regular wards $[6,7]$. Thus, appropriate and timely classification of patients in the ED would aid in the proper allocation of healthcare resources, as well as significantly reduce patient morbidity and mortality.

Sepsis patients can be classified based on various methods, including clinical judgment, scoring systems, and sepsis categories as defined by the Surviving Sepsis Campaign [5]. However, stratification based on scoring systems and clinical judgment has been shown to be superior to classification based on sepsis categories [8]. There are various scoring systems for patients with sepsis that aid not only in determining the severity but also in predicting mortality. These include the Acute Physiology and Chronic Health Evaluation (APACHE) score; Simplified Acute Physiology Score (SAPS); Predisposition, Infection, Response, and Organ dysfunction (PIRO) score; Mortality in Emergency Department Sepsis (MEDS) score; Rapid Emergency Medicine Score; Mortality In Severe Sepsis in the Emergency Department (MISSED) score; Sequential Organ Failure Assessment (SOFA) score; quick SOFA (qSOFA) score; and the Sepsis Patient Evaluation in the Emergency Department (SPEED) score [3,9-17].

Some of these scoring systems are quite comprehensive and were originally designed for usage in the ICU, such as the APACHE score. They require information that may not be readily available to an ED physician, which limits their utility in that setting. Certain scoring systems have been developed for this very purpose in recent years, to guide ED physicians in clinical decision-making and appropriate disposition of patients with sepsis. These scoring systems are simple to calculate, as they utilize limited parameters, yet they can accurately predict mortality. The MEDS score is one of the most widely used scoring systems in the ED. The PIRO score is also a comprehensive tool, and it was devised for usage in the ED. However, EDs in developing countries often have limited infrastructure and budget, and facilities for covariates like band cell count and differential blood cell counts may not be readily available everywhere. The SPEED score utilizes the most fundamental and readily available diagnostics and is simpler than both the MEDS and PIRO scores, making it very useful in the ED setting.

There have been very few studies on the SPEED score in the literature. In light of this, we conducted this study to assess the predictive value of the SPEED score in estimating the 28-day mortality among septic patients who present to the ED. We also compared the predictive value of the SPEED, MEDS, and PIRO scores in estimating the 28-day mortality of sepsis patients presenting to the ED.

\section{Materials And Methods}

This prospective observational study was conducted in the Department of Emergency Medicine at an urban tertiary care hospital. The purpose of this study was to assess the predictive value of the SPEED score for estimating 28-day mortality among patients with sepsis presenting to the ED, as well as compare it to the predictive values of the MEDS and PIRO scores.

We included adult patients who were 18 years of age or older who presented to the ED with suspected or confirmed sepsis and who fulfilled two or more criteria of the qSOFA score in the study. Pregnant females and patients who presented to the ED in cardiac arrest were excluded. Informed written consent was obtained from the patients or their relatives. The study was approved by the Institutional Ethical Committee at AIIMS, Rishikesh (Ref No: AIIMS/IEC/20/381).

\section{Sample size}

On review of the literature, the expected AUC of the SPEED, MEDS, and PIRO scores were $>0.8, \sim 0.75$, and $\sim$.9, respectively for estimating mortality among patients with sepsis who presented to the ED. The sample size calculated for each parameter at a corrected alpha of 0.017 and a conservative expected AUC of 0.75 was 71 (after adjusting for an attrition rate of $20 \%$ due to non-responders) [12,13,17]. A total of 127 patients were included in the study. The calculation was done by package pROC of the R statistical environment [18].

One ROC curve power calculation:

Ncases $=17.49195$ 


\section{Cureus}

Ncontrols $=52.47586$

AUC $=0.75$

Sig. level $=0.016$

Power $=0.8$.

\section{Methods}

Based on the inclusion and exclusion criteria, patients presenting to the ED with suspected or confirmed sepsis were screened for eligibility. If a patient was found to have met the eligibility criteria and once they gave informed written consent, their SPEED, MEDS, and PIRO scores were calculated. Table 1 describes the details related to this. Patients were grouped into four categories based on their SPEED score: $<3$ points, 4- 6 points, 7-9 points, and $>10$ points [17]. Patients were also grouped into five categories according to their MEDS score: $0-4$ points, $5-7$ points, $8-11$ points, $12-14$ points, and $\geqslant 15$ points [13]. Patients were additionally grouped into four categories according to their PIRO score: $<5$ points, 5-9 points, 10-14 points, and 15-19 points [12]. Each patient or their relatives were followed up on day 28, either through hospital records or the telephone, to record final outcomes in terms of survival or mortality.

\section{Parameters}

SPEED score

Immunosuppressed state

Hypotension (systolic blood pressure $<90 \mathrm{mmHg}$ )

Hypothermia (body temperature $<36^{\circ} \mathrm{C}$ )

Hypoxemia (pulse oximetry $<90 \%$ )

Low hematocrit $(<0.38)$

Elevated blood lactate (>2.4 mmol/L)

Pneumonia

Low pH $(<7.35)$

MEDS score

Terminal illness ( $<30$ days)

Hypoxia or tachypnea

Septic shock

Platelet count $<150,000$

Granulocytic bands $>5 \%$

Age $>65$ years

LRTI

Nursing home resident

Altered mental status

PIRO score

Predisposition group

Age $>70$ years

COPD

Cancer

Presence of Foley catheter 


\section{Cureus}

Infection group

Pneumonia 1

CNS: meningitis/encephalitis

Abdomen: UTI

Response group

Bands $>5 \%$

Respiratory rate $>20 /$ minute

Organ dysfunction group

Systolic blood pressure $<90 \mathrm{mmHg}$

$\mathrm{PaO}_{2} / \mathrm{FiO}_{2}<300$

Urine output in the first 2 hours $<30 \mathrm{~mL}$

aPTT $>35$ seconds

Creatinine $>1.8 \mathrm{mg} / \mathrm{dL}$

\section{TABLE 1: Parameters of the SPEED, MEDS, and PIRO scores}

SPEED: Sepsis Patient Evaluation in the Emergency Department; MEDS: Mortality in Emergency Department Sepsis; PIRO: Predisposition, Infection, Response, and Organ Dysfunction; COPD: chronic obstructive pulmonary disease; CLD: chronic liver disease; CNS: central nervous system; UTI: urinary tract infection; LRTI: lower respiratory tract infection; $\mathrm{PaO}_{2}$ : partial pressure of oxygen; $\mathrm{FiO}_{2}$ : fraction of inspired oxygen; aPTT: activated partial thromboplastin time; GCS: Glasgow Coma Scale

\section{Statistical analysis}

The SPSS statistics software version 25 (IBM, Armonk, NY) was used to analyze the data [19]. Categorical variables were represented with numbers and percentages, and continuous variables were presented as mean (SD) and median (IQR) (depending on the distribution of the data after assessing normality via the Shapiro-Wilk test). A chi-squared test or Fisher's exact test was used to analyze the categorical variables. Continuous variables were analyzed with the Wilcoxon-Mann-Whitney U test or a t-test. The receiver operating characteristic (ROC) curves were plotted for each of the three scores, and the area under the ROC curves (AUROC) was computed and analyzed. The level of significance was set at $\mathrm{p}<0.05$.

\section{Results}

After accounting for the inclusion and exclusion criteria, informed consent, and attrition, a total of 127 patients were included in this study. The study population was in the age group of 20-78 years, with a median (IQR) age of 49 (35-63) years. About half of the study population had at least one comorbidity, among which diabetes mellitus $(29 ; 22.8 \%)$, hypertension $(20 ; 15.7 \%)$, and malignancy $(10 ; 7.9 \%)$ were the most common comorbidities; 84 patients $(66.1 \%)$ required intubation and 90 patients $(70.9 \%)$ were admitted to the ICU. By the end of 28 days, 64 patients (50.4\%) had expired. The baseline characteristics of the patients in the study population are summarized in Table 2 .

\begin{tabular}{ll} 
Variable & Values \\
\hline Age, years, median (IQR) & $49.00(35.00$ \\
Gender, $\mathrm{n}(\%)$ & \\
Male & $84(66.1 \%)$ \\
Female & $43(33.9 \%)$ \\
Substance use, $\mathrm{n}(\%)$ & \\
None & $82(64.6 \%)$ \\
Smoking & $13(10.2 \%)$
\end{tabular}




\section{Cureus}

\begin{tabular}{|c|c|}
\hline Alcohol & $3(2.4 \%)$ \\
\hline Tobacco & $3(2.4 \%)$ \\
\hline \multicolumn{2}{|l|}{ Comorbidities, n (\%) } \\
\hline None & $67(52.8 \%)$ \\
\hline Diabetes mellitus & $29(22.8 \%)$ \\
\hline Hypertension & $20(15.7 \%)$ \\
\hline Malignancy & $10(7.9 \%)$ \\
\hline Chronic liver disease & $7(5.5 \%)$ \\
\hline Chronic kidney disease & $7(5.5 \%)$ \\
\hline Chronic obstructive pulmonary disease & $4(3.1 \%)$ \\
\hline Hypothyroidism & $4(3.1 \%)$ \\
\hline Coronary artery disease & $3(2.4 \%)$ \\
\hline Stroke & $3(2.4 \%)$ \\
\hline Bronchial asthma & $2(1.6 \%)$ \\
\hline Pulmonary tuberculosis & $2(1.6 \%)$ \\
\hline \multicolumn{2}{|l|}{ Etiology, n (\%) } \\
\hline Pneumonia & $74(58.3 \%)$ \\
\hline Urinary tract & $40(31.5 \%)$ \\
\hline Blood & $14(11 \%)$ \\
\hline Meningitis & $8(6.3 \%)$ \\
\hline Skin and soft tissue & $4(3.1 \%)$ \\
\hline Intra-abdominal & $1(0.78 \%)$ \\
\hline \multicolumn{2}{|l|}{ General physical examination } \\
\hline Heart rate, $\mathrm{BPM}$, mean $\pm \mathrm{SD}$ & $107.89 \pm 9.64$ \\
\hline Systolic blood pressure, mmHg, median (IQR) & $96.00(80.00-106.00)$ \\
\hline Mean arterial pressure, $\mathrm{mmHg}$, median (IQR) & $76.00(65.00-82.00)$ \\
\hline Diastolic blood pressure, mmHg, median (IQR) & $66.00(58.00-70.00)$ \\
\hline Respiratory rate, CPM, mmHg, median (IQR) & $31.00(24.00-36.00)$ \\
\hline GCS, median (IQR) & $15.00(10.00-15.00)$ \\
\hline \multicolumn{2}{|l|}{ Outcomes, n (\%) } \\
\hline Intubation & $84(66.1 \%)$ \\
\hline ICU admission & $90(70.9 \%)$ \\
\hline HDU admission & $37(29.1 \%)$ \\
\hline Deceased & $64(50.4 \%)$ \\
\hline Alive & $63(49.6 \%)$ \\
\hline
\end{tabular}

TABLE 2: Demographic, clinical parameters, and etiologies of all patients in the study population $(n=127)$

IQR: interquartile range; SD: standard deviation; BPM: beats per minute; CPM: counts per minute; GCS: Glasgow Coma Scale; HDU: high-dependency unit; ICU: intensive care unit 


\section{Cureus}

Table 3 summarizes the distribution of the SPEED, MEDS, and PIRO scores in the study population. The median SPEED, MEDS, and PIRO scores of the study population were 6,8 , and 10, respectively.

\begin{tabular}{|c|c|}
\hline Parameters & Values \\
\hline \multicolumn{2}{|l|}{ SPEED score } \\
\hline Hypoxemia (pulse oximetry <90\%), n (\%) & $79(62.2 \%)$ \\
\hline Pneumonia, n (\%) & $75(59.1 \%)$ \\
\hline Low hematocrit (<0.38), n (\%) & $67(52.8 \%)$ \\
\hline Low pH (<7.35), n (\%) & $64(50.4 \%)$ \\
\hline Elevated blood lactate (>2.4 mmol/L), n (\%) & $61(48.0 \%)$ \\
\hline Hypotension (SBP <90 mmHg), n (\%) & $50(39.4 \%)$ \\
\hline Immunosuppressed state, $\mathrm{n}(\%)$ & $12(9.4 \%)$ \\
\hline Hypothermia (body temperature $<36{ }^{\circ} \mathrm{C}$ ), n (\%) & $7(5.5 \%)$ \\
\hline Total score, median (IQR) & $6(4-9)$ \\
\hline \multicolumn{2}{|l|}{ MEDS score } \\
\hline Hypoxia or tachypnea, n (\%) & $103(81.1 \%)$ \\
\hline LRTI, n (\%) & $74(58.3 \%)$ \\
\hline Granulocytic bands, n (\%) & $59(46.5 \%)$ \\
\hline Platelet count, n (\%) & $55(43.3 \%)$ \\
\hline Altered mental status, n (\%) & $51(40.2 \%)$ \\
\hline Septic shock, n (\%) & $50(39.4 \%)$ \\
\hline Age >65 years, $n(\%)$ & $26(20.5 \%)$ \\
\hline Terminal illness, n (\%) & $1(0.8 \%)$ \\
\hline Nursing home resident, $n$ (\%) & $0(0.0 \%)$ \\
\hline Total score, median (IQR) & $8(5-11)$ \\
\hline \multicolumn{2}{|l|}{ PIRO score } \\
\hline Foley catheter, n (\%) & $75(59.1 \%)$ \\
\hline Age >70 years, $n(\%)$ & $18(14.2 \%)$ \\
\hline Cancer, n (\%) & $12(9.4 \%)$ \\
\hline COPD, n (\%) & $4(3.1 \%)$ \\
\hline CLD, n (\%) & $7(5.5 \%)$ \\
\hline Pneumonia, n (\%) & $75(59.1 \%)$ \\
\hline UTI, n (\%) & $40(31.5 \%)$ \\
\hline Meningitis/encephalitis, n (\%) & $9(7.1 \%)$ \\
\hline RR >20/minute, $n(\%)$ & $103(81.1 \%)$ \\
\hline Bands >5\%, n (\%) & $60(47.2 \%)$ \\
\hline Creatinine $>1.8 \mathrm{mg} / \mathrm{dL}, \mathrm{n}(\%)$ & $53(41.7 \%)$ \\
\hline SBP <90 mmHg, n (\%) & $50(39.4 \%)$ \\
\hline Urine output in the first 2 hours <30 mL, $n(\%)$ & $50(39.4 \%)$ \\
\hline $\mathrm{PaO}_{2} / \mathrm{FiO}_{2}<300, \mathrm{n}(\%)$ & $49(38.6 \%)$ \\
\hline
\end{tabular}




\section{Cureus}

aPTT >35 seconds, $\mathrm{n}$ (\%)

\section{TABLE 3: Distribution of SPEED, MEDS, and PIRO scores in the study population $(n=127)$}

SPEED: Sepsis Patient Evaluation in the Emergency Department; MEDS: Mortality in Emergency Department Sepsis; PIRO: Predisposition, Infection, Response, and Organ Dysfunction; COPD: chronic obstructive pulmonary disease; CLD: chronic liver disease; UTI: urinary tract infection; RR: respiratory rate; SBP: systolic blood pressure; LRTI: lower respiratory tract infection; $\mathrm{PaO}_{2}$ : partial pressure of oxygen; $\mathrm{FiO}_{2}$ : fraction of inspired oxygen; aPTT: activated partial thromboplastin time; GCS: Glasgow Coma Scale; IQR: interquartile range

Table 4 presents a comparison of the SPEED, MEDS, and PIRO scores between survivors and non-survivors. The median SPEED, MEDS, and PIRO scores were higher among non-survivors when compared to survivors. There was a significant difference between the two groups in terms of SPEED score $(\mathrm{W}=408.500, \mathrm{p}<0.001)$, MEDS score $(\mathrm{W}=578.000, \mathrm{p}<0.001)$, and PIRO score $(\mathrm{W}=424.500, \mathrm{p}<0.001)$.

Using point-biserial correlation, the strength of association between the SPEED score and mortality was 0.67 (large effect size), that between MEDS score and mortality was 0.61 (large effect size), and between PIRO score and mortality was 0.28 (medium effect size).

\begin{tabular}{|l|l|l|}
\hline Score & Survivors, median (IQR) & Non-survivors, median (IQR) \\
\hline SPEED score & $4(2.5-6)$ & $9(6.75-11)$ \\
\hline MEDS score & $6(5-8)$ & $11(8.75-14)$ \\
\hline PIRO score & $7(5.5-9)$ & $13(10.75-14)$ \\
\hline
\end{tabular}

\section{TABLE 4: Comparison of median SPEED, MEDS, and PIRO scores between survivors and non-} survivors $(n=127)$

${ }^{1}$ Wilcoxon-Mann-Whitney $U$ test

SPEED: Sepsis Patient Evaluation in the Emergency Department; MEDS: Mortality in Emergency Department Sepsis; PIRO: Predisposition, Infection, Response, and Organ Dysfunction; IQR: interquartile range

As shown in Table 5, there was also a significant difference between survivors and non-survivors in terms of distribution of SPEED score quintiles $\left(\chi^{2}=58.469, \mathrm{p}<0.001\right)$. Using the Cramer's V test, the strength of association between the two variables was 0.68 , signifying a high association. Using the bias-corrected Cramer's V test, the strength of association between the two variables was 0.66 , again signifying a high association. 


\section{Cureus}

\begin{tabular}{|c|c|c|c|c|c|}
\hline \multirow{2}{*}{ SPEED score: category } & \multicolumn{3}{|c|}{ Outcomes, n (\%) } & \multicolumn{2}{|c|}{ Chi-squared test } \\
\hline & Survivors & Non-survivors & Total & $x^{2}$ & P-value \\
\hline$<3$ & 21 (33.3\%) & $1(1.6 \%)$ & $22(17.3 \%)$ & \multirow{5}{*}{58.469} & \multirow{5}{*}{$<0.001$} \\
\hline $4-6$ & 35 (55.6\%) & $15(23.4 \%)$ & $50(39.4 \%)$ & & \\
\hline $7-9$ & $6(9.5 \%)$ & $22(34.4 \%)$ & $28(22.0 \%)$ & & \\
\hline$\geq 10$ & $1(1.6 \%)$ & $26(40.6 \%)$ & $27(21.3 \%)$ & & \\
\hline Total & $63(100.0 \%)$ & $64(100.0 \%)$ & $127(100.0 \%)$ & & \\
\hline
\end{tabular}

TABLE 5: 28-day mortality rate for SPEED score quintiles $(n=127)$

SPEED: Sepsis Patient Evaluation in the Emergency Department

When comparing the individual parameters of the SPEED score between survivors and non-survivors, a significant difference was found between the two groups in the distribution of immunosuppressed state $\left(\chi^{2}\right.$ $=5.752, \mathrm{p}=0.016)$, hypotension $\left(\chi^{2}=10.226, \mathrm{p}=0.001\right)$, hypothermia $\left(\chi^{2}=7.293, \mathrm{p}=0.013\right)$, hypoxemia $\left(\chi^{2}=\right.$ 16.773, $\mathrm{p}<0.001)$, low hematocrit $\left(\chi^{2}=22.141, \mathrm{p}<0.001\right)$, elevated blood lactate $\left(\chi^{2}=18.966, \mathrm{p}<0.001\right)$, and low $\mathrm{pH}\left(\chi^{2}=17.390, \mathrm{p}<0.001\right)$ parameters. However, there was no significant difference between the two groups in terms of distribution of pneumonia $\left(\chi^{2}=1.338, \mathrm{p}=0.247\right)$ (Table 6 ).

Using bias-corrected Cramer's V test, the strength of the association between immunosuppressed state and mortality was 0.19 (low association), that between hypotension and mortality was 0.27 (low association), between hypothermia and mortality was 0.22 (low association), between hypoxemia and mortality was 0.35 (moderate association), between hematocrit and mortality was 0.41 (moderate association), between blood lactate and mortality was 0.38 (moderate association), and between $\mathrm{pH}$ and mortality was 0.36 (moderate association).

\begin{tabular}{|c|c|c|c|}
\hline SPEED score parameters & Survivors, n (\%) & Non-survivors, n (\%) & P-value \\
\hline Immunosuppressed state & $2(3.2 \%)$ & $10(15.6 \%)$ & $0.016^{1}$ \\
\hline Hypotension (systolic blood pressure $<90$ mmHg) & $16(25.4 \%)$ & $34(53.1 \%)$ & $0.001^{1}$ \\
\hline Hypothermia (body temperature $<36{ }^{\circ} \mathrm{C}$ ) & $0(0.0 \%)$ & $7(10.9 \%)$ & $0.013^{2}$ \\
\hline Hypoxemia (pulse oximetry <90\%) & $28(44.4 \%)$ & $51(79.7 \%)$ & $<0.001^{1}$ \\
\hline Low hematocrit $(<0.38)$ & $20(31.7 \%)$ & $47(73.4 \%)$ & $<0.001^{1}$ \\
\hline Elevated blood lactate (>2.4 mmol/L) & $18(28.6 \%)$ & $43(67.2 \%)$ & $<0.001^{1}$ \\
\hline Pneumonia & $34(54.0 \%)$ & $41(64.1 \%)$ & $0.247^{1}$ \\
\hline Low pH (<7.35) & $20(31.7 \%)$ & $44(68.8 \%)$ & $<0.001^{1}$ \\
\hline
\end{tabular}

TABLE 6: Group comparison of SPEED score parameters between survivors and non-survivors (n = 127)

${ }^{1}$ Chi-squared test. ${ }^{2}$ Fisher's exact test

SPEED: Sepsis Patient Evaluation in the Emergency Department

The area under the receiver operating characteristic curve (AUROC) for SPEED score for predicting 28-day mortality among patients with sepsis was 0.899 (95\% CI: 0.847-0.951), thereby demonstrating good diagnostic performance (Figure 1). It was statistically significant $(\mathrm{p}<0.001)$. At a cut-off of $\geqslant 7$, the SPEED 


\section{Cureus}

score predicts 28 -day mortality with a sensitivity of $75 \%$ and a specificity of $89 \%$ (Table 7 ).

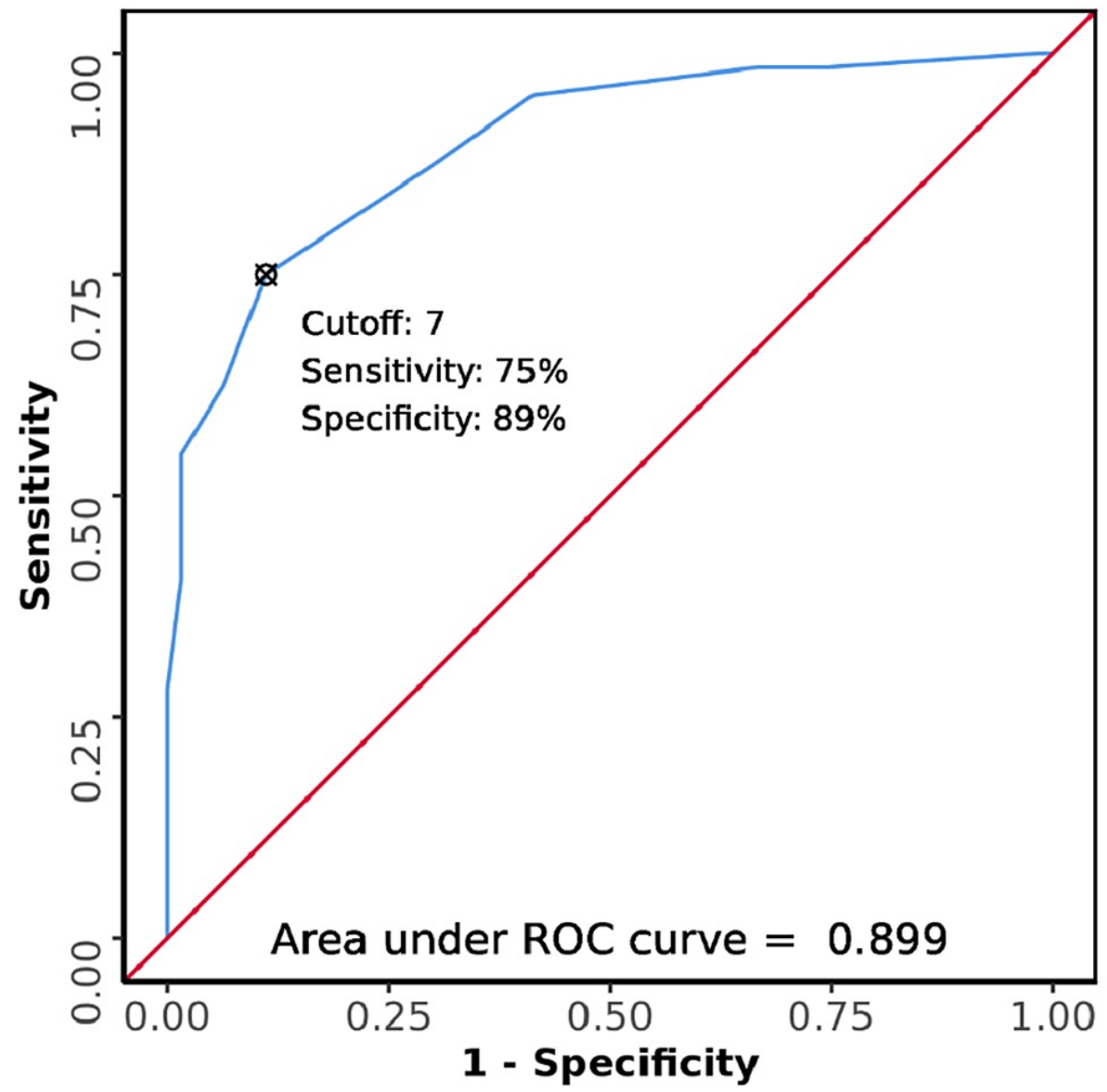

FIGURE 1: ROC curve of SPEED score for 28-day mortality among patients with sepsis who presented to the emergency department ROC: receiver operating characteristic; SPEED: Sepsis Patient Evaluation in the Emergency Department

\begin{tabular}{|c|c|c|c|}
\hline \multirow[t]{2}{*}{ Parameter } & SPEED score & MEDS score & PIRO score \\
\hline & Value $(95 \% \mathrm{Cl})$ & Value $(95 \% \mathrm{Cl})$ & Value $(95 \% \mathrm{CI})$ \\
\hline AUROC & $0.899(0.847-0.951)$ & $0.857(0.793-0.92)$ & $0.895(0.838-0.951)$ \\
\hline Cut-off (p-value) & $\geq 7(<0.001)$ & $\geq 10(<0.001)$ & $\geq 10(<0.001)$ \\
\hline Sensitivity & $75.0 \%(63-85)$ & $71.9 \%(59-82)$ & $87.5 \%(77-94)$ \\
\hline Specificity & $88.9 \%(78-95)$ & $84.1 \%(73-92)$ & $77.8 \%(66-87)$ \\
\hline Positive predictive value & $87.3 \%(76-95)$ & $82.1 \%(70-91)$ & $80.0 \%(69-89)$ \\
\hline Negative predictive value & $77.8 \%(66-87)$ & $74.6 \%(63-84)$ & $86.0 \%(74-94)$ \\
\hline
\end{tabular}

TABLE 7: ROC curve analysis showing diagnostic performance of SPEED score, MEDS score, and PIRO score in predicting 28-day mortality among patients with sepsis $(n=127)$

AUROC: area under the receiver operating characteristic curve; Cl: confidence interval; SPEED: Sepsis Patient Evaluation in the Emergency Department; MEDS: Mortality in Emergency Department Sepsis; PIRO: Predisposition, Infection, Response, and Organ Dysfunction 


\section{Cureus}

The AUROC for MEDS score for predicting 28-day mortality among patients with sepsis was 0.857 (95\% CI: 0.793-0.92), thereby demonstrating good diagnostic performance (Figure 2). It was statistically significant ( $\mathrm{p}$ $<0.001)$. At a cut-off of $\geqslant 10$, the MEDS score predicts 28 -day mortality with a sensitivity of $72 \%$ and a specificity of $84 \%$ (Table 7 ).

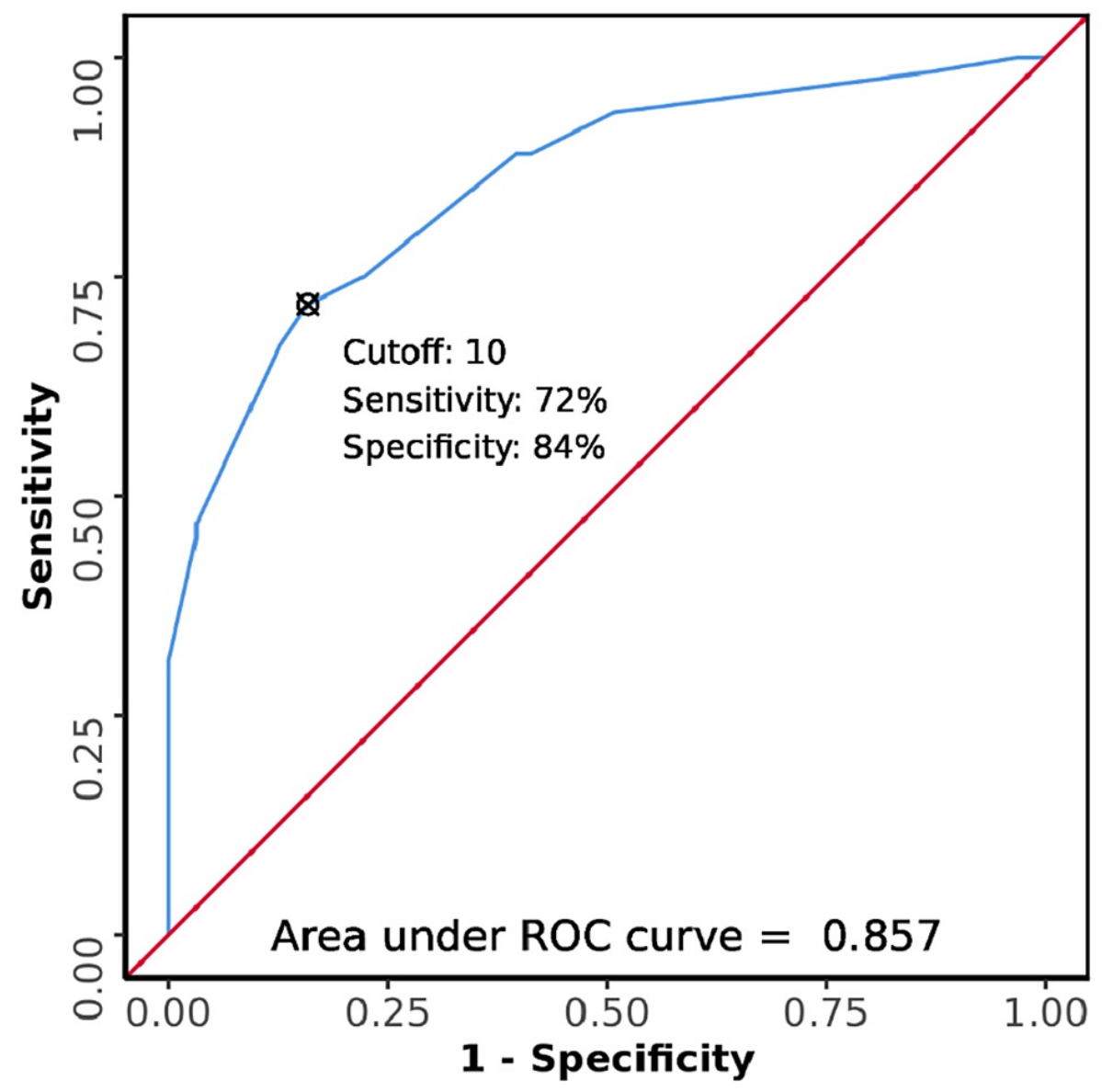

FIGURE 2: ROC curve of MEDS score for 28-day mortality among patients with sepsis who presented to the emergency department

ROC: receiver operating characteristic; MEDS: Mortality in Emergency Department Sepsis

The AUROC for the PIRO score for predicting 28-day mortality among patients with sepsis was 0.895 (95\% CI: 0.838-0.951), thus demonstrating good diagnostic performance (Figure 3). It was statistically significant $(\mathrm{p}<0.001)$. At a cut-off of $\geqslant 10$, the PIRO score predicts 28 -day mortality with a sensitivity of $88 \%$ and a specificity of $78 \%$ (Table 7 ). 


\section{Cureus}

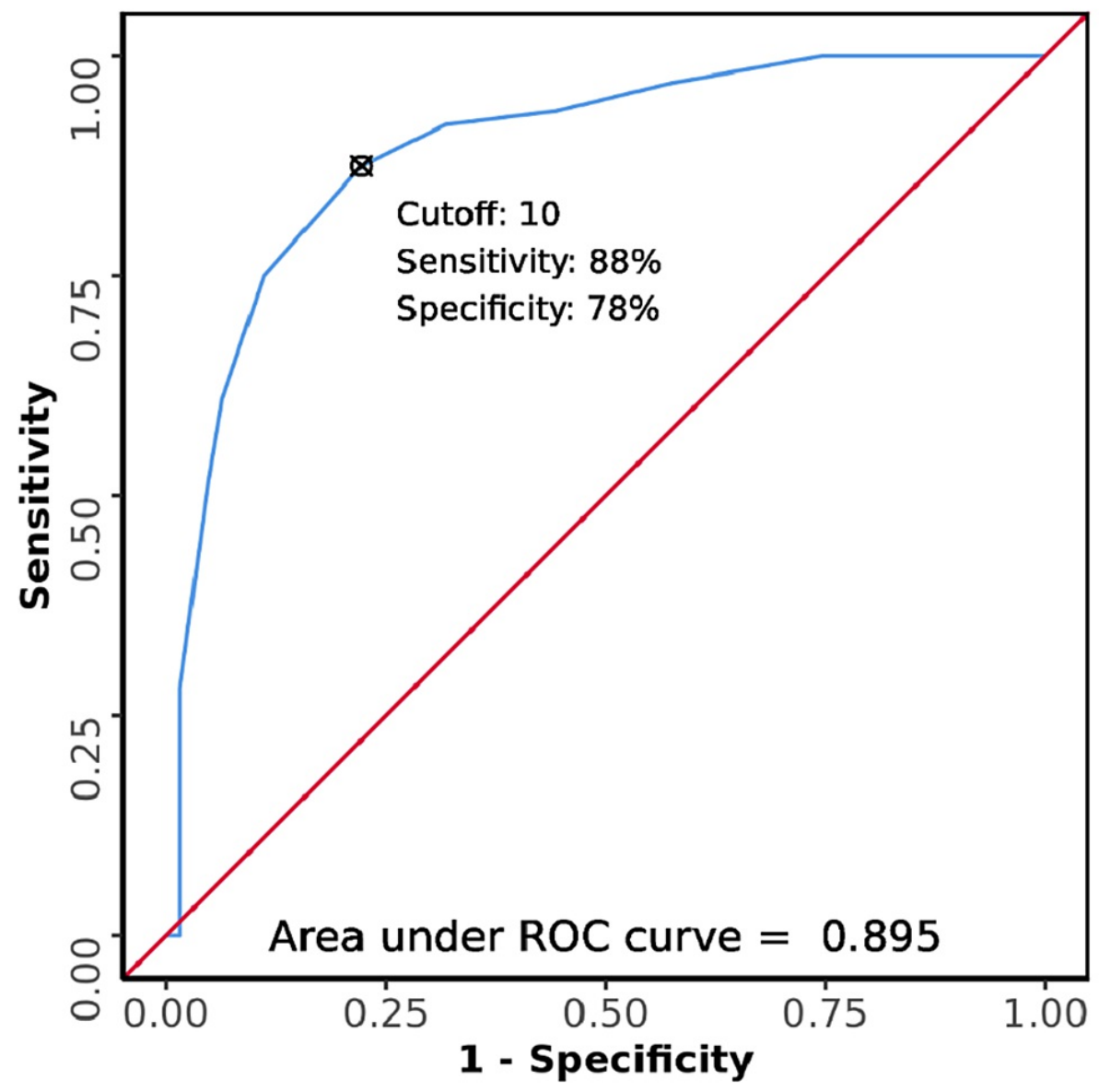

FIGURE 3: ROC curve of PIRO score for 28-day mortality among patients with sepsis who presented to the emergency department

ROC: receiver operating characteristic; PIRO: Predisposition, Infection, Response, and Organ Dysfunction

Figure 4 shows the diagnostic performances of the SPEED, MEDS, and PIRO scores in predicting 28-day mortality among patients with sepsis. There was no significant difference between the diagnostic performance of the SPEED score and MEDS score (DeLong test $\mathrm{p}=0.182$ ), nor between the SPEED score and PIRO score (DeLong test $\mathrm{p}=0.904$ ). There was also no significant difference in the diagnostic performance between the PIRO score and MEDS score (DeLong test $\mathrm{p}=0.203$ ). 


\section{Cureus}

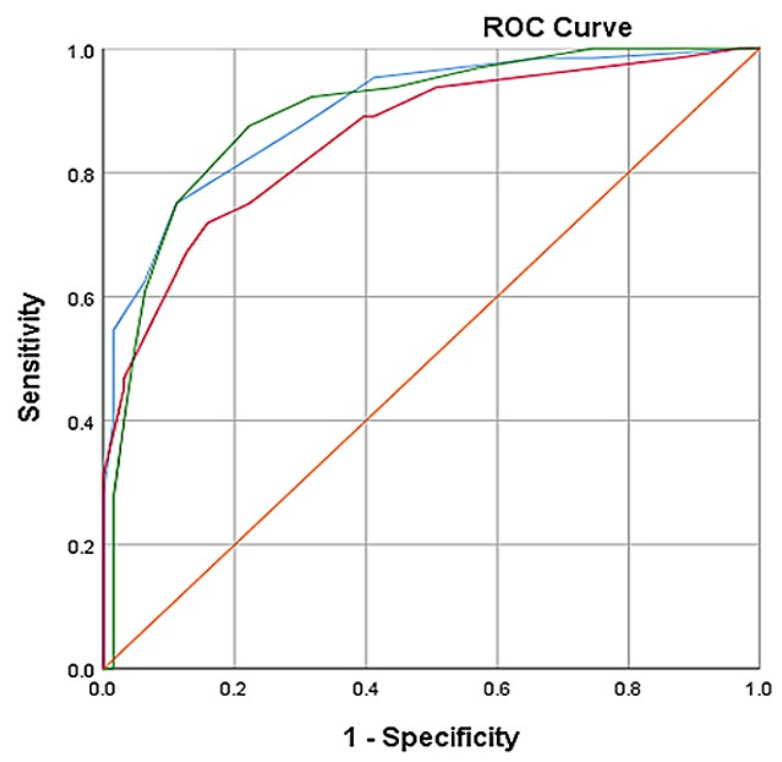

Source of the Curve

Diagonal segments are produced by ties.

FIGURE 4: ROC curves of the SPEED score, MEDS score, and PIRO score for 28-day mortality among patients with sepsis who presented to the emergency department

ROC: receiver operating characteristic; SPEED: Sepsis Patient Evaluation in the Emergency Department; MEDS Mortality in Emergency Department Sepsis; PIRO: Predisposition, Infection, Response, and Organ Dysfunction

\section{Discussion}

In this prospective observational study, the 28 -day mortality was found to be $50.4 \%$. Similar to our findings, the study by Todi et al. showed a 28 -day mortality rate of $57.6 \%$ in patients with severe sepsis [20]. The study by Darba and Marsà reported similar findings, with a case fatality rate of $42.9 \%$ in patients with septic shock [21]. In the study by Chatterjee et al., the 28-day mortality rate of patients with severe sepsis was reported to be $62.8 \%$, which is considerably higher than in the current study [22]. This difference can be attributed to their study design: sepsis was defined in their study as the presence of both infection and systemic inflammatory response syndrome (SIRS), while severe sepsis was defined as the presence of sepsis fulfilling at least one criterion for organ dysfunction. This study included patients with sepsis as defined by the qSOFA criteria. Thus, the study by Chatterjee et al. included a much sicker population. Pneumonia was the most common site of infection in our study, followed by urinary tract infections. The respiratory tract has been reported as the most common site of infection among patients with sepsis in several previous studies [22-26].

All the three scores assessed in the study (SPEED, MEDS, and PIRO) were found to be a significant predictor of 28 -day mortality among patients with sepsis $(\mathrm{p}<0.001)$. The mortality rate was higher in patients with a higher SPEED score, as shown in Table 5. These findings are similar to those by Bewersdorf et al. and Elbaih et al. [17,27]. The SPEED score is a composite score comprising eight parameters: immunosuppressed state, hypotension, hypothermia, hypoxemia, low hematocrit, pneumonia, elevated blood lactate, and acidosis. Among these eight parameters, all except pneumonia were found to be significantly associated with mortality. Multiple studies conducted previously have reported that the presence of an immunosuppressed state, hypotension, hypothermia, hypoxemia, low hematocrit, elevated blood lactate, and acidosis were significantly associated with mortality among patients with sepsis [28-44]. Pneumonia was not found to be significantly associated with mortality among patients with sepsis in this study $(p=0.247)$. Similar findings have been reported in the studies by Xie et al., Huang et al., and Wang et al. [24,25,28]. Bewersdorf et al. have reported that all eight parameters of the SPEED score were significantly associated with mortality [17]. However, only two parameters - hypotension and hypoxemia - were reported as significantly associated with mortality by Elbaih et al. [27].

The AUROC for the SPEED score in this study was 0.899 (95\% CI: 0.847-0.951). The cut-off value of the SPEED score obtained was $\geqslant 7$. At this value, the SPEED score predicted mortality with a sensitivity of $75 \%$, specificity of $89 \%$, a positive predictive value of $87.3 \%$, and a negative predictive value of $77.8 \%$. In the study by Bewersdorf et al., the AUROC for the SPEED score was 0.83 (95\% CI: 0.79-0.88) in the derivation set and 0.80 (95\% CI: 0.73-0.87) in the validation set [17]. In the study by Elbaih et al., the AUROC for SPEED score was 0.87 (95\% CI: 0.788-0.963) [27]. Although none of these studies reported a cut-off value for the SPEED 
The AUROC for the MEDS score was 0.857 (95\% CI: 0.793-0.92). The cut-off value of the MEDS score obtained was $\geqslant 10$. At this value, the MEDS score predicted mortality with a sensitivity of $71.9 \%$, specificity of $84.1 \%$, a positive predictive value of $82.1 \%$, and a negative predictive value of $74.6 \%$. In the study by Shapiro et al., the AUROC for the MEDS score for predicting mortality in the derivation set was 0.82 , while it was 0.78 in the validation set [13]. In a meta-analysis by Zhang et al., the AUROC for the MEDS score was 0.83 (95\% CI: $0.80-0.86$ ), with a pooled sensitivity of $79 \%$ and specificity of $74 \%$ to predict mortality. A substantial variation was found in the cut-off value of the MEDS score used to predict mortality, with values ranging from 7 to 14.5 . However, MEDS scores of 8-12 were most frequently chosen as the optimal cut-off values, with 8 being the most frequent value [45].

The value of the AUROC obtained in this study was found to be quite similar to the above-mentioned studies. The cut-off score obtained in this study was 10, which is higher than what was reported in the meta-analysis by Zhang et al. This was due to choosing a value that had a higher specificity as compared to sensitivity. The hospital care center in this study receives extremely ill patients, and hence specificity is of greater importance than sensitivity in this setting.

The AUROC for the PIRO score was 0.895 (95\% CI: 0.838-0.951) in this study, with an obtained cut-off value of $\geqslant 10$. At this value, the PIRO score predicted mortality with a sensitivity of $87.5 \%$, specificity of $77.8 \%$, a positive predictive value of $80.0 \%$, and a negative predictive value of $86.0 \%$. The value of AUROC obtained is quite similar to that by Rathour et al. In their study, the AUROC of the PIRO score for predicting mortality was reported as 0.94 (95\% CI: 0.900-0.971) [12]. However, the study by Caramello et al. reported the AUROC for PIRO score as 0.765 (95\% CI: 0.71-0.82) for 30-day mortality and 0.754 (0.701-0.806) for 60-day mortality [46]. None of these studies reported a cut-off value for the PIRO score. In the current study, the SPEED score was found to be the best predictor of 28-day mortality among patients with sepsis, with an AUROC of 0.899 . This was followed by the PIRO score with an AUROC of 0.895, and the MEDS score with an AUROC of 0.857 . No statistically significant difference was found between the diagnostic performance of the three scores. Similar findings have been reported in the studies by Bewersdorf et al. and Elbaih et al. [17,27].

The MEDS score is used widely in the ED for risk stratification of patients with sepsis. The PIRO score is a detailed classification system involving multiple parameters, which was designed for use in the ED. Both MEDS and PIRO scores require the band cell percentage for calculation, which may not be available to ED physicians everywhere. The PIRO score additionally requires activated partial thromboplastin time (aPTT) values for calculation, which again may not be readily available to ED physicians. The SPEED score is a much simpler score, and it utilizes parameters that are readily available even in resource-limited settings. Since the diagnostic performances of all these scores are quite similar, the SPEED score has the advantage of being the simple and easy choice to reliably predict mortality.

\section{Limitations}

This study has some limitations. Firstly, the sample size was small; hence, a larger, multi-center study would be required before applying these results to the general population. Secondly, very few patients satisfied certain parameters of the individual scores, such as hypothermia and nursing home residency. Thus, the relevance of these individual parameters in predicting mortality among patients with sepsis could not be accurately determined. Thirdly, the likely infective agents responsible for sepsis in this study's healthcare setting may be different from other healthcare settings, and this could confound the utility of the scores. Additionally, death due to causes other than infection could not be entirely excluded. Finally, these findings cannot be extrapolated to children and pregnant females.

\section{Conclusions}

The SPEED score is a simple and handy tool that relies on parameters readily available at the point-of-care in the ED, and it is also suitable for usage in resource-limited settings. In this study, there was no significant difference between the diagnostic performances of the SPEED, MEDS, and PIRO scores in predicting the 28day mortality among patients with sepsis. Hence, the SPEED score can be utilized as one of the early risk stratification methods for patients with sepsis in the ED. This can help in allocating healthcare resources rationally in terms of timely admission of patients to an appropriate level of care, ultimately resulting in a significant impact on patient outcomes.

\section{Additional Information \\ Disclosures}

Human subjects: Consent was obtained or waived by all participants in this study. All India Institute of Medical Sciences, Rishikesh issued approval AIIMS/IEC/20/381. Animal subjects: All authors have confirmed that this study did not involve animal subjects or tissue. Conflicts of interest: In compliance with the ICMJE uniform disclosure form, all authors declare the following: Payment/services info: All authors have declared that no financial support was received from any organization for the submitted work. Financial relationships: All authors have declared that they have no financial relationships at present or 
within the previous three years with any organizations that might have an interest in the submitted work. Other relationships: All authors have declared that there are no other relationships or activities that could appear to have influenced the submitted work.

\section{Acknowledgements}

The authors would like to thank Enago (www.enago.com) for the English language review.

\section{References}

1. Rudd KE, Johnson SC, Agesa KM, et al.: Global, regional, and national sepsis incidence and mortality, 19902017: analysis for the Global Burden of Disease Study. Lancet. 2020, 395:200-11. 10.1016/S01406736(19)32989-7

2. Badrinath K, Shekhar M, Sreelakshmi M, et al.: Comparison of various severity assessment scoring systems in patients with sepsis in a tertiary care teaching hospital. Indian J Crit Care Med. 2018, 22:842-5. 10.4103/ijccm.IJCCM_322_18

3. Singer M, Deutschman CS, Seymour CW, et al.: The Third International Consensus Definitions for Sepsis and Septic Shock (Sepsis-3). JAMA. 2016, 315:801-10. 10.1001/jama.2016.0287

4. Rivers E, Nguyen B, Havstad S, et al.: Early goal-directed therapy in the treatment of severe sepsis and septic shock. N Engl J Med. 2001, 345:1368-77. 10.1056/NEJMoa010307

5. Dellinger RP, Levy MM, Rhodes A, et al.: Surviving Sepsis Campaign: international guidelines for management of severe sepsis and septic shock, 2012. Intensive Care Med. 2013, 39:165-228. 10.1007/s00134-012-2769-8

6. Kennedy M, Joyce N, Howell MD, Lawrence Mottley J, Shapiro NI: Identifying infected emergency department patients admitted to the hospital ward at risk of clinical deterioration and intensive care unit transfer. Acad Emerg Med. 2010, 17:1080-5. 10.1111/j.1553-2712.2010.00872.x

7. Lundberg JS, Perl TM, Wiblin T, Costigan MD, Dawson J, Nettleman MD, Wenzel RP: Septic shock: an analysis of outcomes for patients with onset on hospital wards versus intensive care units. Crit Care Med. 1998, 26:1020-4. 10.1097/00003246-199806000-00019

8. de Groot B, Lameijer J, de Deckere ER, Vis A: The prognostic performance of the predisposition, infection, response and organ failure (PIRO) classification in high-risk and low-risk emergency department sepsis populations: comparison with clinical judgement and sepsis category. Emerg Med J. 2014, 31:292-300. 10.1136/emermed-2012-202165

9. Zimmerman JE, Kramer AA, McNair DS, Malila FM: Acute Physiology and Chronic Health Evaluation (APACHE) IV: hospital mortality assessment for today's critically ill patients. Crit Care Med. 2006, 34:1297310. 10.1097/01.CCM.0000215112.84523.F0

10. Metnitz PG, Moreno RP, Almeida E, et al.: SAPS 3--from evaluation of the patient to evaluation of the intensive care unit. Part 1: objectives, methods and cohort description. Intensive Care Med. 2005, 31:133644. 10.1007/s00134-005-2762-6

11. Moreno RP, Metnitz PG, Almeida E, et al.: SAPS 3--from evaluation of the patient to evaluation of the intensive care unit. Part 2: development of a prognostic model for hospital mortality at ICU admission. Intensive Care Med. 2005, 31:1345-55. 10.1007/s00134-005-2763-5

12. Rathour S, Kumar S, Hadda V, Bhalla A, Sharma N, Varma S: PIRO concept: staging of sepsis . J Postgrad Med. 2015, 61:235-42. 10.4103/0022-3859.166511

13. Shapiro NI, Wolfe RE, Moore RB, Smith E, Burdick E, Bates DW: Mortality in Emergency Department Sepsis (MEDS) score: a prospectively derived and validated clinical prediction rule. Crit Care Med. 2003, 31:670-5. 10.1097/01.CCM.0000054867.01688.D1

14. Olsson T, Terent A, Lind L: Rapid Emergency Medicine score: a new prognostic tool for in-hospital mortality in nonsurgical emergency department patients. J Intern Med. 2004, 255:579-87. 10.1111/j.13652796.2004.01321.x

15. Sivayoham N, Rhodes A, Cecconi M: The MISSED score, a new scoring system to predict Mortality in Severe Sepsis in the Emergency Department: a derivation and validation study. Eur J Emerg Med. 2014, 21:30-6. 10.1097/MEJ.0b013e328364a8d4

16. Vincent JL, Moreno R, Takala J, et al.: The SOFA (Sepsis-related Organ Failure Assessment) score to describe organ dysfunction/failure. On behalf of the Working Group on Sepsis-Related Problems of the European Society of Intensive Care Medicine. Intensive Care Med. 1996, 22:707-10. 10.1007/BF01709751

17. Bewersdorf JP, Hautmann O, Kofink D, Abdul Khalil A, Zainal Abidin I, Loch A: The SPEED (sepsis patient evaluation in the emergency department) score: a risk stratification and outcome prediction tool. Eur J Emerg Med. 2017, 24:170-5. 10.1097/MEJ.0000000000000344

18. Robin X, Turck N, Hainard A, Tiberti N, Lisacek F, Sanchez JC, Müller M: pROC: an open-source package for $\mathrm{R}$ and S+ to analyze and compare ROC curves. BMC Bioinformatics. 2011, 12:77. 10.1186/1471-2105-12-77

19. IBM Corp. Released 2017 IBM SPSS Statistics for Windows, Version 25.0 . (2017). Accessed: February 24, 2022: https://www.ibm.com/docs/en/spss-statistics/25.0.0.

20. Todi S, Chatterjee S, Bhattacharyya M: Epidemiology of severe sepsis in India. Crit Care. 2007, 11:P65. 10.1186/cc5225

21. Darbà J, Marsà A: Epidemiology, management and costs of sepsis in Spain (2008-2017): a retrospective multicentre study. Curr Med Res Opin. 2020, 36:1089-95. 10.1080/03007995.2020.1760809

22. Chatterjee S, Bhattacharya M, Todi SK: Epidemiology of adult-population sepsis in India: a single center 5 year experience. Indian J Crit Care Med. 2017, 21:573-7. 10.4103/ijccm.IJCCM_240_17

23. Mayr FB, Yende S, Angus DC: Epidemiology of severe sepsis. Virulence. 2014, 5:4-11. 10.4161/viru.27372

24. Xie J, Wang H, Kang Y, et al.: The epidemiology of sepsis in Chinese ICUs: a national cross-sectional survey . Crit Care Med. 2020, 48:e209-18. 10.1097/CCM.0000000000004155

25. Huang CT, Tsai YJ, Tsai PR, Yu CJ, Ko WJ: Epidemiology and outcome of severe sepsis and septic shock in surgical intensive care units in northern Taiwan. Medicine (Baltimore). 2015, 94:e2136. 
10.1097/MD.0000000000002136

26. Abe T, Ogura H, Kushimoto $\mathrm{S}$, et al.: Variations in infection sites and mortality rates among patients in intensive care units with severe sepsis and septic shock in Japan. J Intensive Care. 2019, 7:28. 10.1186/s40560-019-0383-3

27. Elbaih AH, Elsayed ZM, Ahmed RM, Abd-Elwahed SA: Sepsis patient evaluation emergency department (SPEED) score \& mortality in emergency department sepsis (MEDS) score in predicting 28-day mortality of emergency sepsis patients. Chin J Traumatol. 2019, 22:316-22. 10.1016/j.cjtee.2019.10.004

28. Wang JY, Chen YX, Guo SB, Mei X, Yang P: Predictive performance of quick Sepsis-related Organ Failure Assessment for mortality and ICU admission in patients with infection at the ED. Am J Emerg Med. 2016, 34:1788-93. 10.1016/j.ajem.2016.06.015

29. Maheshwari K, Nathanson BH, Munson SH, et al.: The relationship between ICU hypotension and inhospital mortality and morbidity in septic patients. Intensive Care Med. 2018, 44:857-67. 10.1007/s00134018-5218-5

30. Marchick MR, Kline JA, Jones AE: The significance of non-sustained hypotension in emergency department patients with sepsis. Intensive Care Med. 2009, 35:1261-4. 10.1007/s00134-009-1448-X

31. Poutsiaka DD, Davidson LE, Kahn KL, Bates DW, Snydman DR, Hibberd PL: Risk factors for death after sepsis in patients immunosuppressed before the onset of sepsis. Scand J Infect Dis. 2009, 41:469-79. 10.1080/00365540902962756

32. Jamme M, Daviaud F, Charpentier J, et al.: Time course of septic shock in immunocompromised and nonimmunocompromised patients. Crit Care Med. 2017, 45:2031-9. 10.1097/CCM.0000000000002722

33. Rumbus Z, Matics R, Hegyi P, et al.: Fever is associated with reduced, hypothermia with increased mortality in septic patients: a meta-analysis of clinical trials. PLoS One. 2017, 12:e0170152. 10.1371/journal.pone.0170152

34. Zhang Z, Ji X: Quadratic function between arterial partial oxygen pressure and mortality risk in sepsis patients: an interaction with simplified acute physiology score. Sci Rep. 2016, 6:35133. 10.1038/srep35133

35. Kho AN, Hui S, Kesterson JG, McDonald CJ: Which observations from the complete blood cell count predict mortality for hospitalized patients?. J Hosp Med. 2007, 2:5-12. 10.1002/jhm.143

36. Nguyen HB: Lactate in the critically ill patients: an outcome marker with the times . Crit Care. 2011, 15:1016. 10.1186/cc10531

37. Kushimoto S, Akaishi S, Sato T, et al.: Lactate, a useful marker for disease mortality and severity but an unreliable marker of tissue hypoxia/hypoperfusion in critically ill patients. Acute Med Surg. 2016, 3:293-7. 10.1002/ams2.207

38. Filho RR, Rocha LL, Corrêa TD, Pessoa CM, Colombo G, Assuncao MS: Blood lactate levels cutoff and mortality prediction in sepsis-time for a reappraisal? A retrospective cohort study. Shock. 2016, 46:480-5. 10.1097/SHK.0000000000000667

39. Liu Z, Meng Z, Li Y, Zhao J, Wu S, Gou S, Wu H: Prognostic accuracy of the serum lactate level, the SOFA score and the qSOFA score for mortality among adults with sepsis. Scand J Trauma Resusc Emerg Med. 2019, 27:51. 10.1186/s13049-019-0609-3

40. Villar J, Short JH, Lighthall G: Lactate predicts both short- and long-term mortality in patients with and without sepsis. Infect Dis (Auckl). 2019, 12:1178633719862776. 10.1177/1178633719862776

41. Gunnerson KJ, Saul M, He S, Kellum JA: Lactate versus non-lactate metabolic acidosis: a retrospective outcome evaluation of critically ill patients. Crit Care. 2006, 10:R22. 10.1186/cc3987

42. Khosravani H, Shahpori R, Stelfox HT, Kirkpatrick AW, Laupland KB: Occurrence and adverse effect on outcome of hyperlactatemia in the critically ill. Crit Care. 2009, 13:R90. 10.1186/cc7918

43. Wernly B, Heramvand N, Masyuk M, et al.: Acidosis predicts mortality independently from hyperlactatemia in patients with sepsis. Eur J Intern Med. 2020, 76:76-81. 10.1016/j.ejim.2020.02.027

44. Noritomi DT, Soriano FG, Kellum JA, Cappi SB, Biselli PJ, Libório AB, Park M: Metabolic acidosis in patients with severe sepsis and septic shock: a longitudinal quantitative study. Crit Care Med. 2009, 37:2733-9. 10.1097/ccm.0b013e3181a59165

45. Zhang G, Zhang K, Zheng X, Cui W, Hong Y, Zhang Z: Performance of the MEDS score in predicting mortality among emergency department patients with a suspected infection: a meta-analysis. Emerg Med J. 2020, 37:232-9. 10.1136/emermed-2019-208901

46. Caramello V, Macciotta A, Beux V, De Salve AV, Ricceri F, Boccuzzi A: Validation of the Predisposition Infection Response Organ (PIRO) dysfunction score for the prognostic stratification of patients with sepsis in the emergency department (EPUB ahead of print). Med Intensiva (Engl Ed). 2020, 10.1016/j.medin.2020.04.022 